\title{
Risk of Symptomatic Intracerebral Hemorrhage in Patients Treated with Intra-Arterial Thrombolysis
}

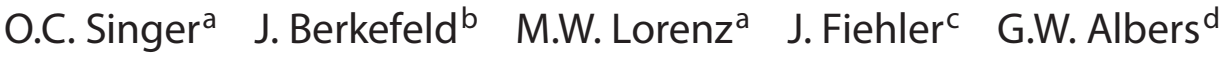 \\ M.G. Lansberg ${ }^{d} \quad$ A. Kastrup ${ }^{e}{ }^{f} \quad$ A. Rovirag $\quad$ D.S. Liebeskind ${ }^{\text {h }} \quad$ A. Gass ${ }^{i}$ \\ C. Rosso ${ }^{j} \quad$ L. Derex ${ }^{k} \quad$ J.S. Kim' T. Neumann-Haefelin ${ }^{a}$ \\ for the MR Stroke Study Group Investigators
}

\begin{abstract}
${ }^{a}$ Klinik für Neurologie and ${ }^{b}$ Institut für Neuroradiologie, Universitätsklinik, Goethe-Universität, Frankfurt, ${ }^{\mathrm{C}}$ Neuroradiologie, Universitätsklinik Eppendorf, Hamburg, Germany; ${ }^{\mathrm{d}}$ Department of Neurology, Stanford University Medical Center, Stanford, Calif., USA; ${ }^{\mathrm{e}}$ Neurologische Klinik, Universitätsklinikum, Jena,

${ }^{\mathrm{f}}$ Neurologische Klinik, Universitätsklinikum, Göttingen, Germany; ${ }^{9}$ Department of Radiology, Hospital Universitari Vall d'Hebron, Barcelona, Spain; hUCLA Stroke Center, University of California, Los Angeles, Calif., USA; 'Neurologische Klinik, Universitätsklinikum, Mannheim, Germany; 'Urgences Cérébro-Vasculaires, Salpêtrière, AP-HP, Paris, ${ }^{k}$ Cerebrovascular Disease Center, Creatis UMR CNRS 5515, Inserm U 630, University of Lyon, Lyon, France; 'Department of Neurology, University of Ulsan, Asan Medical Center, Seoul, Korea
\end{abstract}

\section{Key Words}

Stroke, acute - Thrombolysis, Intra-arterial · Hemorrhage, intracerebral

\begin{abstract}
Background: In intra-arterial (IA) thrombolysis trials, higher rates of symptomatic intracerebral haemorrhage $(\mathrm{sICH})$ were found than in trials with intravenous (IV) recombinant tissue plasminogen activator (tPA); this observation could have been due to the inclusion of more severely affected patients in IA thrombolysis trials. In the present study, we investigated the rate of sICH in IA and combined IV + IA thrombolysis versus IV thrombolysis after adjusting for differences in clinical and MRI parameters. Methods: In this multicenter study, we systematically analyzed data from 645 patients with anterior-circulation strokes treated with either IV or IA thrombolysis within $6 \mathrm{~h}$ following symptom onset. Thrombolytic regimens included (1) IV tPA treatment $(n=536)$ and (2) IA
\end{abstract}

treatment with either tPA or urokinase $(n=74)$ or (3) combined IV + IA treatment with either tPA or urokinase $(n=35)$. Results: 44 (6.8\%) patients developed sICH. sICH patients had significantly higher scores on the National Institutes of Health Stroke Scale (NIHSS) at admission and pretreatment DWI lesions. The sICH risk was 5.2\% $(n=28)$ in IV thrombolysis, which is significantly lower than in IA $(12.5 \%, \mathrm{n}=9)$ or IV + IA thrombolysis $(20 \%, n=7)$. In a binary logistic regression

Disclosures: The study was initiated by the MR Stroke Study group without dedicated funding. Local data sampling was supported by different funding sources. Bundesministerium für Bildung und Wissenschaft within the Kompetenznetzwerk Schlaganfall (Stroke Imaging Network); Stanford, Calif.: National Institute for Neurological Disorders and Stroke RO1 NS39325 and K23 NS051372; Los Angeles, Calif.: NIH Grant 5P50NS044378-03; Paris: PHRC AOM 03008 EVALUSINV, and Seoul: Brain Research Center of the 21st Century Frontier Research Program funded by the Ministry of Science and Technology of Korea (M103KV010010 06K2201 01010).

\section{KARGER}

(C) 2009 S. Karger AG, Basel

Fax +41613061234 E-Mail karger@karger.ch www.karger.com www.karger.com/ced
Dr. Oliver C. Singer, MD

Department of Neurology, J.W. Goethe University Frankfurt am Main

Schleusenweg 2-16, DE-60528 Frankfurt/Main (Germany)

Tel. +49696301 5942, Fax +496963014498

E-Mail o.singer@em.uni-frankfurt.de 
analysis including age, NIHSS score, time to thrombolysis, initial diffusion weighted imaging lesion size, mode of thrombolytic treatment and thrombolytic agent, the mode of thrombolytic treatment remained an independent predictor for sICH. The odds ratio for IA or IV + IA versus IV treatment was 3.466 (1.19-10.01, 95\% Cl, p < 0.05). Conclusion: In this series, IA and IV + IA thrombolysis is associated with an increased sICH risk as compared to IV thrombolysis, and this risk is independent of differences in baseline parameters such as age, initial NIHSS score or pretreatment lesion size.

Copyright $\odot 2009$ S. Karger AG, Basel

\section{Introduction}

Intravenous (IV) thrombolysis with recombinant tissue plasminogen activator (tPA) has been proven safe and effective for the treatment of acute ischemic stroke [1]. Nevertheless, in many patients recanalization of the occluded artery is not achieved despite treatment, particularly in patients with proximal occlusions [1-3]. Intra-arterial (IA) thrombolysis, on the other hand, or a combination of IV and IA (IV + IA) thrombolysis ('bridging therapy') has the potential to achieve substantially higher rates of recanalization [4], and several studies have proven its feasibility in acute stroke [4-6]. Furthermore, IA thrombolysis offers several other potential advantages such as direct vessel monitoring, and the possibility of additional mechanical clot manipulation. The drawback of longer time-to-treatment intervals by IA thrombolysis may be resolved by initiation of immediate IV thrombolysis followed by IA treatment. A recent observational study in selected stroke patients (hyperdense media sign on CT) comparing IV and IA thrombolysis found an improved outcome after 3 months in patients undergoing IA thrombolysis while mortality was similar in both treatment groups [7].

However, both treatment strategies share the risk of potentially life-threatening symptomatic intracerebral hemorrhage (sICH). Robust data on sICH rates in IV thrombolysis are available from several large randomized trials [8] as well as from observational stroke registries [19] indicating that the sICH risk in IV thrombolysis ranges between 2 and $8 \%$ (depending on the definition of sICH). However, data on sICH rates in IA or IV + IA thrombolysis are limited to relatively small studies. In the largest controlled IA thrombolysis study [4], sICH occurred in $10 \%$ of 108 patients; smaller studies on combined IV + IA thrombolysis reported sICH rates of 6.3, 9.9 and $11.8 \%$, respectively $[5,6,9]$. Other observational studies on IA or combined IV + IA thrombolysis in clinical routine reported sICH rates of up to $17.9 \%$ [10].

The reason for the apparently higher rate of $\mathrm{sICH}$ in IA or IV + IA thrombolysis trials is not entirely clear, but it could to be due to the higher proportion of severely affected patients in IA thrombolysis studies. It is also possible that IA thrombolysis itself is associated with an increased sICH risk, possibly due to the relatively high concentration of thrombolytic agent at the site of application. Finally, differences in sICH definitions have likely contributed to the variability of sICH rates among studies.

The aim of this large multicenter analysis was to compare sICH rates in a large cohort of patients treated with IV and IA or combined IV + IA thrombolysis using a uniform sICH definition. To determine the independent association between route of tPA administration and sICH risk, a multivariate analysis was performed to adjust for imbalances in baseline variables.

\section{Patients and Methods}

\section{Patients}

This retrospective multicenter analysis was performed based on systematically and prospectively collected data provided by 10 well-established academic stroke centers (Barcelona, Frankfurt, Hamburg, Jena, Los Angeles, Calif., Lyon, Mannheim, Paris, Stanford, Calif., for the DEFUSE investigators [24], and Seoul). The study was conducted as an initiative of the MR Stroke Study group, which is an international group of stroke physicians who meet regularly with the aim to coordinate and standardize MRI research in acute stroke. Each of the centers participating in the current study uses standardized MRI protocols for acute stroke patients, approved by all local ethics committees. Clinical and imaging data of the majority of patients included in the present analysis have been published before by the MR Stroke Study Group [11-13].

Patients were included in the current analysis, if (1) they presented with anterior circulation stroke, (2) thrombolytic therapy was administered within $6 \mathrm{~h}$ of symptom onset, and (3) an MRI including diffusion-weighted imaging (DWI) was performed before initiation of treatment. For the primary analysis, symptomatic ICH was defined, as in the NINDS trial (with only minimal modifications), as CT- (or MRI-) documented hemorrhage occurring within $36 \mathrm{~h}$ of treatment onset and was temporally related to deterioration in the patient's clinical condition in the clinician's judgment, associated with a worsening of at least 1 point on the National Institutes of Health Stroke Scale (NIHSS) [1]. CT and MRI scans of sICH patients were read centrally for hemorrhage classification according to ECASS criteria [14]. Additionally, ICH volume was estimated using the $\mathrm{ABC} / 2$ formula [15]. The following clinical variables associated with sICH were obtained for each patient: age, NIHSS score on admission and time between symptom onset and first-line thrombolytic treatment (categorized $<3$, 3-6 h). Furthermore, the NIHSS score at the time of clinical deterioration or on routine follow-up (days 2-7) was obtained. An 
NIHSS score of 42 points was assigned to patients who deceased within the follow-up period.

\section{Thrombolytic Treatment}

Thrombolytic treatment regimens included: (1) standard IV tPA therapy with a dosage of $0.9 \mathrm{mg} / \mathrm{kg}(\mathrm{n}=536),(2)$ local IA thrombolysis $(n=74)$ using either tPA $(n=37)$ or urokinase $(n=$ 37) with or without additional mechanical clot manipulation (excluding the use of special devices for clot removal such as the MERCI retriever and the Penumbra system), (3) IV + IA thrombolysis $(n=35)$ using either tPA $(n=27)$ or a combination of tPA and urokinase $(n=8)$ with or without additional mechanical clot manipulation. No heparin was administered within the $24 \mathrm{~h}$ after thrombolysis, except for low amounts of heparin during angiography, at the discretion of the treating interventionalist.

\section{MRI Data Acquisition and Analysis}

MRI scans were performed on 1.5-tesla scanners, all equipped with echo-planar imaging (EPI) data acquisition capabilities. Stroke protocols were not entirely uniform in the 10 participating centers, but all included EPI DWI. No predefined MRI patterns (such as DWI/PWI mismatch) were required for inclusion in the study. Pretreatment DWI lesion volumes were measured by the participating stroke centers using locally available software.

\section{Statistical Analysis}

As the variables age, initial NIHSS score and initial DWI volume were not normally distributed (Kolmogorov-Smirnov test), the Mann-Whitney test was used to test for statistically significant differences between groups. To control for multiple tests when comparing three groups pair-wise, we applied the closing test procedure with the Kruskal-Wallis test as global test [16]. Binary logistic regression was used to determine independent risk factors for sICH. The influential variable of interest 'route of thrombolysis administration' was coded with two dummy variables (IV thrombolysis yes/no and IA thrombolysis yes/no) as a combined treatment strategy ('bridging') was applied in a subcohort. In multivariate models, we adjusted for the confounders 'age', 'NIHSS score at presentation', 'DWI lesion volume', 'time to thrombolysis' and 'used thrombolytics' (rTPA or urokinase). Results were considered statistically significant at the $5 \%$ level. Unless otherwise stated, all values are given as median (25th, 75th percentiles). For illustration purposes (fig. 1), IA and IV + IA patients were grouped together.

\section{Results}

Data from 645 patients meeting the inclusion criteria were analyzed. 536 patients received IV thrombolysis, 109 patients were treated either with IA or combined IV + IA thrombolysis. Overall, 44 (6.8\%) patients developed sICH. Out of those 44 patients, 30 had parenchymal hematoma grade 2,11 patients had parenchymal hematoma grade 1, 2 patients had hemorrhagic infarction grade 2 and 1 patient had hemorrhagic infarction grade $1 . \mathrm{Pa}$ tients with subsequent sICH had higher NIHSS scores at

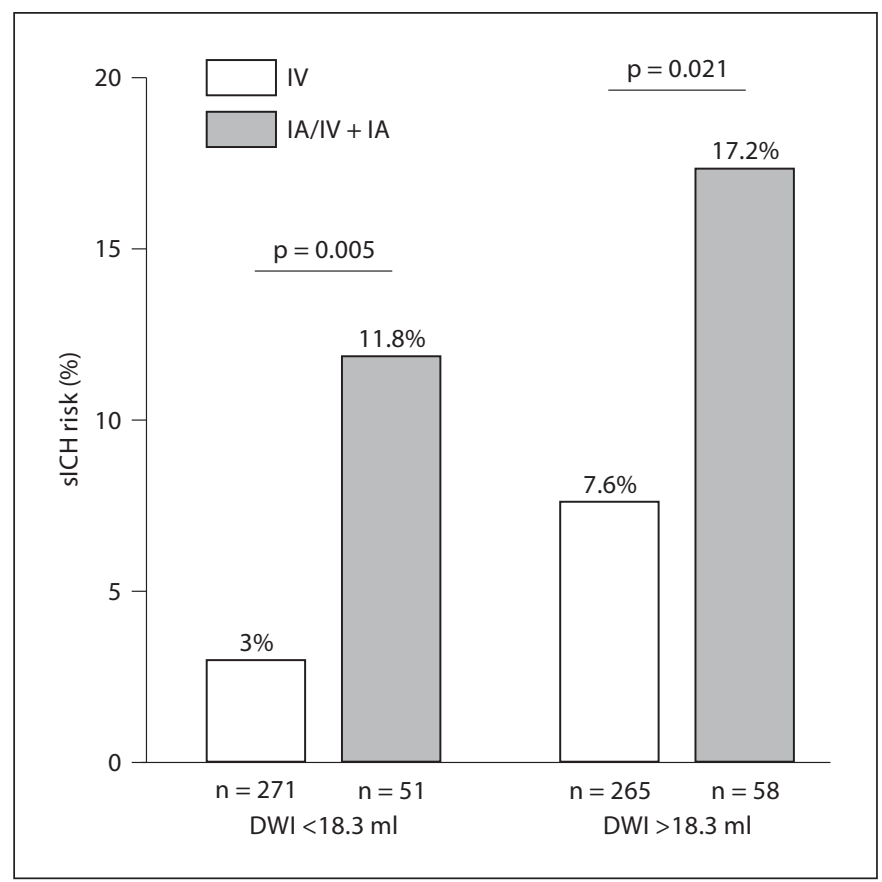

Fig. 1. sICH rates in patients treated with IV as compared to patients treated with IA/IV + IA thrombolysis depending on the initial DWI lesion volume. DWI lesion groups were compiled according to the median DWI lesion volume of all patients $(18.3 \mathrm{ml})$ in a small-lesion group $(<18.3 \mathrm{ml})$ and a large-lesion group $(>18.3$ $\mathrm{ml})$. This graph illustrates that the sICH risk is increased in the IA/IV + IA treatment group independently of the initial DWI lesion size.

Table 1. Basic clinical and imaging data

\begin{tabular}{lllr}
\hline Parameter & $\begin{array}{l}\text { No sICH } \\
(\mathrm{n}=601)\end{array}$ & $\begin{array}{l}\text { sICH } \\
(\mathrm{n}=44)\end{array}$ & $\begin{array}{r}\mathrm{p} \\
\text { value }^{1}\end{array}$ \\
\hline Age, years & $69(57-76)$ & $71(61-77)$ & 0.11 \\
Sex, male & $54 \%$ & $56 \%$ & 0.821 \\
NIHSS at presentation & $13(9,17)$ & $17(12,20)$ & 0.003 \\
DWI volume, ml & $18(7,42)$ & $44(15,93)$ & $<0.001$ \\
Time to thrombolysis $>3 \mathrm{~h}$ & $49 \%$ & $55 \%$ & 0.504 \\
\hline
\end{tabular}

${ }^{1}$ Mann-Whitney U test.

admission and had larger DWI lesions prior to thrombolytic therapy as compared to patients without sICH (table 1). Delayed time to treatment (3-6 vs. 0-3 h after symptom onset) was not associated with a significantly increased sICH risk in any of the treatment routes (IV, IA 
Table 2. sICH rates in patients treated with IV, IA or IV + IA thrombolysis

\begin{tabular}{lrc}
\hline Thrombolytic therapy & \multicolumn{1}{l}{$\mathrm{n}$} & \multicolumn{1}{l}{ sICH } \\
\hline IV & 536 & $28(5.2 \%)^{\mathrm{a}, \mathrm{b}}$ \\
IA & 74 & $9(12.2 \%)^{\mathrm{a}, \mathrm{c}}$ \\
IV + IA & 35 & $7(20 \%)^{\mathrm{b}, \mathrm{c}}$ \\
\hline
\end{tabular}

The Kruskal-Wallis test revealed significant differences for sICH risk between the three groups ( $p<0.01$ ). The Mann-Whitney $U$ test was used for comparison between two groups.

${ }^{\mathrm{a}} \mathrm{p}<0.05$ between IV and IA thrombolysis; ${ }^{\mathrm{b}} \mathrm{p}<0.01$ between IV and combined IV + IA thrombolysis; ${ }^{\mathrm{c}}$ n.s. $(\mathrm{p}=0.283)$ between IA and combined IV + IA thrombolysis .

Table 3. Multivariate analysis of sICH prediction $(n=634)$

\begin{tabular}{llll}
\hline Variable & OR & $95 \%$ CI & p value \\
\hline Age & 1.019 & $0.99-1.05$ & 0.133 \\
NIHSS score at presentation & 1.059 & $0.99-1.12$ & 0.06 \\
DWI volume, $10 \mathrm{ml}^{1}$ & 1.07 & $1.02-1.12$ & 0.006 \\
Time to thrombolysis $^{2}$ & 1.337 & $0.68-2.63$ & 0.398 \\
Used thrombolytics $^{\text {IA thrombolysis }}{ }^{3}$ & 1.495 & $0.66-3.39$ & 0.335 \\
IV thrombolysis $^{4}$ & 3.466 & $1.19-10.01$ & 0.023 \\
& 2.231 & $0.68-2.63$ & 0.398 \\
\hline
\end{tabular}

${ }^{1}$ OR given for each 10 -ml increase in DWI lesion volume.

${ }^{2}$ Categorized, $0-3$ versus 3-6 h.

${ }^{3}$ IA/IV + IA thrombolysis versus IV thrombolysis.

${ }^{4}$ IV thrombolysis versus IA/IV + IA thrombolysis.

or IV + IA treatment). The overall sICH rate (IV, IA or IV + IA treatment $)$ was $6.3 \%(\mathrm{n}=20)$ in patients treated within $3 \mathrm{~h}$ of symptom onset and $7.6 \%(\mathrm{n}=24)$ in patients treated within 3-6 h $(\mathrm{p}=0.50)$.

The sICH rate was significantly higher in patients treated with IA $(12.2 \%, \mathrm{n}=9)$ or combined IV + IA thrombolysis $(20 \%, \mathrm{n}=7)$ as compared to patients treated with IV thrombolysis $(5.2 \%, \mathrm{n}=28$ ) (table 2). After adjusting for age, initial NIHSS score, time to thrombolytic treatment, initial DWI lesion, the thrombolytic agent used (tPA vs. urokinase) in a multivariate model $(\mathrm{n}=634)$, the route of tPA administration remained significantly associated with an increased sICH risk. Patients treated with IA or IV + IA thrombolysis were more likely to develop sICH than patients treated with IV thrombolysis (odds ratio, OR 3.466; 95\% CI 1.19-10.01, $p<0.05$ ) (table 3). The initial DWI lesion volume was independently associated with sICH as reported previously (OR 1.07 for a $10-\mathrm{ml}$ increase in DWI volume; 95\% CI $1.02-1.12, \mathrm{p}<0.01)[13,17]$. Despite a clear trend towards significance, the NIHSS score at presentation was not an independent risk factor for $\mathrm{sICH}$ in this analysis.

Figure 1 illustrates that even in patients with small DWI lesions $(<18.3 \mathrm{ml}$, the median DWI lesion volume of the study population) IA/IV + IA treatment was associated with a 3.9-fold higher risk for sICH; in patients with large DWI lesions, the risk for sICH associated with IA administration was increased 2.3-fold. This finding is in accordance with the fact that DWI lesion size and mode of thrombolytic treatment are independent risk factors for sICH.

For the primary analysis, sICH criteria of the NINDStPA trial were applied. Using stricter criteria of sICH resulted in lower total rates of sICH whereas the sICH rate in patients treated IA or IV + IA remained constantly higher as compared to IV-treated patients (table 4).

\section{Discussion}

This retrospective multicenter analysis is the first large study directly comparing sICH rates in MR-guided IV versus IA or IV + IA thrombolysis in clinical routine. We found an increased sICH risk in IA- and IV + IA-treated patients, which could not solely be explained by differences in clinical and imaging baseline parameters.

The hemorrhage rate in patients treated by IV thrombolysis in this study was comparable to the rates reported in the large IV thrombolysis trials $[1,8,18]$. The fact, that sICH rates are similar (or even lower) in communitybased registries or in observational studies [19] as compared to the large randomized trials argues for the successful transfer of IV thrombolytic therapy to the clinical routine. Nevertheless, the efficacy of IV tPA therapy is limited, mainly due to failure to achieve timely recanalization, indicating a clear need for improved treatment regimens.

IA thrombolysis has not been evaluated as rigorously as IV thrombolytic therapy and remains less well standardized. While IA thrombolysis has proven to be more efficacious in achieving vessel patency, sICH rates in IA thrombolysis vary substantially (between 5.5 and 17.9\%) $[4-7,10,20,21]$, and it is likely that differences in treatment protocols such as choice and dosage of the thrombolytic agent, its mode of application, the use of additional mechanical clot manipulation or co-medication have a 
Table 4. sICH rates using different definitions of sICH

\begin{tabular}{lllll}
\hline sICH definition & \multicolumn{4}{l}{ Symptomatic hemorrhage, $\mathrm{n}$} \\
\cline { 2 - 5 } & total & IV & IA & IV + IA \\
\hline Any ICH and NIHSS score difference $\geq 1^{1}$ & $44(6.8)$ & $28(5.2)$ & $9(12.2)$ & $7(20)$ \\
PH1/PH2 and NIHSS score difference $\geq 1$ & $41(6.4)$ & $26(4.9)$ & $9(12.2)$ & $6(17.1)$ \\
Any ICH and NIHSS score difference $\geq 4^{2}$ & $27(4.2)$ & $20(3.7)$ & $5(6.8)$ & $2(5.7)$ \\
PH2 and NIHSS score difference $\geq 4^{3}$ & $21(3.3)$ & $16(3.0)$ & $4(5.4)$ & $1(2.9)$ \\
\hline
\end{tabular}

Figures in parentheses are percentages. $\mathrm{PH} 1, \mathrm{PH} 2=$ Parenchymal hematoma grade 1 or 2 .

${ }^{1}$ NINDS criteria. ${ }^{2}$ PROACT II criteria. ${ }^{3}$ SITS-MOST criteria.

Table 5. sICH rates in IA thrombolysis trials

\begin{tabular}{|c|c|c|c|c|c|c|c|c|c|}
\hline & $\begin{array}{l}\text { Present } \\
\text { study }\end{array}$ & $\begin{array}{l}\text { PRO- } \\
\text { ACT I [25] }\end{array}$ & $\begin{array}{l}\text { PROACT II } \\
{[4]}\end{array}$ & IMS I [5] & IMS II [9] & EMS [6] & $\begin{array}{l}\text { Kidwell } \\
\text { et al. [10] }\end{array}$ & $\begin{array}{l}\text { Mattle } \\
\text { et al. [7] }\end{array}$ & $\begin{array}{l}\text { Brekenfeld } \\
\text { et al. [22] }\end{array}$ \\
\hline Patients, IA treatment, $\mathrm{n}$ & 109 & 26 & 108 & $80^{1}$ & $81^{2}$ & 35 & 89 & & \\
\hline Thrombolytic agent & $\begin{array}{l}\text { r-tPA, } \\
\text { urokinase }\end{array}$ & $\begin{array}{l}\text { pro- } \\
\text { urokinase }\end{array}$ & $\begin{array}{l}\text { pro- } \\
\text { urokinase }\end{array}$ & $\mathrm{r}-\mathrm{tPA}$ & $\mathrm{r}-\mathrm{tPA}$ & $\mathrm{r}-\mathrm{tPA}$ & $\begin{array}{l}\text { r-tPA, } \\
\text { urokinase }\end{array}$ & urokinase & urokinase \\
\hline Mode of treatment & $\mathrm{IA}, \mathrm{IV}+\mathrm{IA}$ & IA & IA & IV, IV + IA & IV, IV + IA & $\begin{array}{l}\text { IV + IA, } \\
\text { placebo + } \\
\text { IA }\end{array}$ & IA, IV + IA & IA & IA \\
\hline $\begin{array}{l}\text { Time window } \\
\text { sICH definition used }\end{array}$ & $0-6 \mathrm{~h}$ & $0-6 \mathrm{~h}$ & $0-6 \mathrm{~h}$ & $0-3 \mathrm{~h}$ & $0-3 \mathrm{~h}$ & $0-3 \mathrm{~h}$ & $0-6(-24 \mathrm{~h})$ & $0-6 \mathrm{~h}$ & $0-6 \mathrm{~h}$ \\
\hline $\begin{array}{l}\text { Any ICH and NIHSS score } \\
\text { difference } \geq 1^{3}\end{array}$ & $16(14.7 \%)$ & & & & & & $16(18 \%)$ & & \\
\hline $\begin{array}{l}\text { Any ICH and NIHSS score } \\
\text { difference } \geq 4^{4}\end{array}$ & & & $11(10.2 \%)$ & & & & & $4(7 \%)$ & \\
\hline Any ICH + 'clinical deterioration' & & $4(15.4 \%)$ & & $5(6.3 \%)$ & $8(9.9 \%)$ & $3(8.6 \%)$ & & & \\
\hline $\mathrm{PH}$ and NIHSS score difference $\geq 4$ & & & & & & & & & $14(4.8 \%)$ \\
\hline
\end{tabular}

substantial impact. However, direct comparison of sICH rates between different IA thrombolysis trials is strongly hampered by the use of various sICH definitions and analysis procedures: while some studies report sICH data separately for IA-treated patients, others also include patients with IV thrombolysis who were screened for IA treatment but were not eligible due to angiographic exclusion criteria, prohibiting a clear estimate of sICH rates associated with the IA treatment approach $[5,9]$. A recent retrospective study on 294 patients undergoing IA thrombolysis using urokinase found a low sICH rate of $4.8 \%$ [22]. However, the authors used a strict definition of sICH (evidence of space-occupying parenchymal hematoma and NHISS deterioration of $\geq 4$ points). Applying the same sICH criteria to IV thrombolysis translates into a substantially lower sICH rate in IV thrombolysis of $1.7 \%$ [19]. Table 5 gives an overview of sICH rates in larger IA thrombolysis trials or observational studies.

The present analysis does not support the common notion that the increased sICH risk of IA thrombolysis is solely attributable to basic patient-related factors such as advanced age or clinical severity of stroke or larger pretreatment ischemic lesions. The reasons for the increased sICH risk remain to be clarified. From a pathophysiological point of view, the relatively high concentration of thrombolytic agent at the site of IA application may play an important role, leading to a variety of processes including a stronger activation of proteases at the blood- 
brain barrier than with IV treatment. Nevertheless, other procedural issues, including additional mechanical manipulation, limited experience with IA thrombolysis as compared to IV thrombolysis, influence of comedication and lack of standardization of IA thrombolysis, have to be considered and deserve special interest in the future.

The present analysis has several limitations. Patients were treated in the participating centers according to local treatment protocols including heterogeneous comedication. Due to the observational nature of the study, no randomization to the treatment groups was performed and the criteria for systemic or IA thrombolysis were not equal within the centers. While the dosage of tPA in IV thrombolysis was standardized, the dosage used for IA or IA + IV tPA varied between the centers. There is a clear imbalance in the numbers of patients in the three treatment groups. However, this imbalance towards IV treatment reflects clinical practice, even in experienced stroke centers with high turnover. For reasons of statistical power, we used the sICH definition applied in the NINDS tPA trial, which has been criticized for a potential overestimation of sICH (in contrast to clinical deterioration e.g. due to reocclusion). Nevertheless, the relative differences in sICH rates in both treatment groups remained similar, even when stricter definitions of sICH were applied. The fact that all but 1 IA treated patient with sICH had a parenchymal hematoma ( $\mathrm{PH} 1$ or $\mathrm{PH} 2)$ argues strongly against a relevant overestimation of hemorrhage rates due to residual angiographic contrast agent that could potentially result in hyperdensity on follow-up CT scan. We cannot exclude, that other patient-related factors, potentially influencing the ICH risk (i.e. hypertension, glucose level, platelet count, albuminuria or preexistent antiplatelet therapy) contribute to the higher sICH rates in IA or IV + IA treated patients, since these parameters were not systematically assessed in all our patients [17, 23]. The present study was not designed to evaluate the efficacy of IA thrombolytic treatment, explaining the lack of data on recanalization rates and long-term followup.

In conclusion, we found an excess rate of sICH in patients undergoing IA and IV + IA thrombolysis as compared to patients treated with IV thrombolysis that was independent of baseline differences in clinical and imaging parameters. Nevertheless, in light of a suboptimal standard therapy - IV thrombolysis -, interventional recanalization strategies for acute stroke remain promising treatment options, and further research concerning technical aspects as well as optimal patient selection is warranted.

\section{Appendix}

MR-Stroke Study Group

Co-chairs: Geoffrey A. Donnan, Stephen M. Davis.

\section{Contributing Members}

Germany: University Hospital of Frankfurt (Tobias Neumann-Haefelin, Oliver C. Singer, Silke Hoelig, Matthias W. Lorenz, Marek C. Humpich, Matthias Sitzer, Helmuth Steinmetz, Bernard Yan, Joachim Berkefeld). University Hospital of Hamburg Eppendorf (Jens Fiehler, Joachim Roether, Thomas Kucinski, Hermann Zeumer, Goetz Thomalla). University Hospital of Jena (Andreas Kastrup). University Hospital of Mannheim (Achim Gass, Olivera Lecei).

France: Salpêtrière, Paris (Yves Samson, Charlotte Rosso, Sandrine Deltour, Sophie Crozier, Anne Léger, Pr. Jacques Chiras and his neuroradiology team). Hôpital neurologique, Lyon (Laurent Derex, Norbert Nighoghoshian, Marc Hermier).

Spain: Hospital Universitari Vall d'Hebron, Barcelona (Alex Rovira, Raquel Delgado, Carlos Molina, Jose Alvarez-Sabin).

United States and Canada: UCLA Stroke Center, University of California, Los Angeles, Calif. (David S. Liebeskind, Jeffrey L. Saver, Jeffrey R. Alger, Latisha K. Ali, Brian H. Buck, Gary R. Duckwiler, Reza Jahan, Doojin Kim, Bruce Ovbiagele, Noriko Salamon, Nerses Sanossian, Sidney Starkman, Paul M. Vespa, J. Pablo Villablanca, Fernando Viñuela). Stanford Stroke Center, Stanford University (Gregory W. Albers, Roland Bammer, Anna Caulfield Finley, Scott Hamilton, Wataru Kakuda, Stephanie Kemp, Maarten G. Lansberg, Michael Marks, Michael Moseley, Neil E. Schwartz, Vincent N. Thijs, Christine A.C. Wijman). UPMC Stroke Institute and Department of Neurology, University of Pittsburgh, Pittsburg, Pa. (Lawrence Wechsler). Beth Israel Deaconess Medical Center and Harvard Medical School, Boston, Mass. (Gottfried Schlaug). Department of Neurology, University of Utah, Salt Lake City, Utah (Elaine Skalabrin). Division of Neurology, University of Alberta, Edmonton (William Coplin).

Korea: Asian Medical Center, Seoul (Jong S. Kim, Dong-Wha Kang, Sun U. Kwon).

References
The NINDS study group: Tissue plasminogen activator for acute ischemic stroke. N Engl J Med 1995;333:1581-1587.

2 Labiche LA, Al-Senani F, Wojner AW, Grotta JC, Malkoff M, Alexandrov AV: Is the benefit of early recanalization sustained at 3 months? A prospective cohort study. Stroke 2003;34: 695-698.

-3 Alexandrov AV, Grotta JC: Arterial reocclusion in stroke patients treated with intravenous tissue plasminogen activator. Neurology 2002;59:862-867.

-4 Furlan A, Higashida R, Wechsler L, Gent M, Rowley H, Kase C, Pessin M, Ahuja A, Callahan F, Clark WM, Silver F, Rivera F: Intra-arterial prourokinase for acute ischemic stroke. The PROACT II study: a randomized controlled trial. Prolyse in acute cerebral thromboembolism. JAMA 1999;282:2003-2011. 
5 Combined intravenous and intra-arterial recanalization for acute ischemic stroke: the Interventional Management of Stroke Study. Stroke 2004;35:904-911.

-6 Lewandowski CA, Frankel M, Tomsick TA, Broderick J, Frey J, Clark W, Starkman S, Grotta J, Spilker J, Khoury J, Brott T: Combined intravenous and intra-arterial r-tPA versus intra-arterial therapy of acute ischemic stroke: emergency management of stroke (EMS) bridging trial. Stroke 1999;30: 2598-2605.

$\checkmark 7$ Mattle HP, Arnold M, Georgiadis D, Baumann C, Nedeltchev K, Benninger D, Remonda L, von Budingen C, Diana A, Pangalu A, Schroth G, Baumgartner RW: Comparison of intraarterial and intravenous thrombolysis for ischemic stroke with hyperdense middle cerebral artery sign. Stroke 2008;39: 379-383.

-8 Hacke W, Donnan G, Fieschi C, Kaste M, von Kummer R, BroderickJP, Brott T, Frankel M, Grotta JC, Haley EC Jr, Kwiatkowski T, Levine SR, Lewandowski C, Lu M, Lyden P, Marler JR, Patel S, Tilley BC, Albers G, Bluhmki E, Wilhelm M, Hamilton S: Association of outcome with early stroke treatment: pooled analysis of ATLANTIS, ECASS, and NINDS rt-PA stroke trials. Lancet $2004 ; 363: 768-774$.

9 The Interventional Management of Stroke (IMS) II Study. Stroke 2007;38:2127-2135.

$>10$ Kidwell CS, Saver JL, Carneado J, Sayre J, Starkman S, Duckwiler G, Gobin YP, Jahan R, Vespa P, Villablanca JP, Liebeskind DS, Vinuela F: Predictors of hemorrhagic transformation in patients receiving intra-arterial thrombolysis. Stroke 2002;33:717-724.

-11 Fiehler J, Albers GW, Boulanger JM, Derex L, Gass A, Hjort N, Kim JS, Liebeskind DS, Neumann-Haefelin T, Pedraza S, Rother J, Rothwell P, Rovira A, Schellinger PD, Trenkler J: Bleeding risk analysis in stroke imaging before thrombolysis (BRASIL): pooled analysis of $\mathrm{t}_{2}{ }^{*}$-weighted magnetic resonance imaging data from 570 patients. Stroke 2007; 38:2738-2744.
12 Neumann-Haefelin T, Hoelig S, Berkefeld J, Fiehler J, Gass A, Humpich M, Kastrup A, Kucinski T, Lecei O, Liebeskind DS, Rother J, Rosso C, Samson Y, Saver JL, Yan B: Leukoaraiosis is a risk factor for symptomatic intracerebral hemorrhage after thrombolysis for acute stroke. Stroke 2006;37:2463-2466.

13 Singer OC, Humpich MC, Fiehler J, Albers GW, Lansberg MG, Kastrup A, Rovira A, Liebeskind DS, Gass A, Rosso C, Derex L, Kim JS, Neumann-Haefelin T: Risk for symptomatic intracerebral hemorrhage after thrombolysis assessed by diffusion-weighted magnetic resonance imaging. Ann Neuro 2008;63:52-60.

14 Larrue V, von Kummer R, del Zoppo G, Bluhmki E: Hemorrhagic transformation in acute ischemic stroke. Potential contributing factors in the European cooperative acute stroke study. Stroke 1997;28:957-960.

15 Kothari RU, Brott T, Broderick JP, Barsan WG, Sauerbeck LR, Zuccarello M, Khoury J: The ABCs of measuring intracerebral hemorrhage volumes. Stroke 1996;27:1304-1305.

16 Marcus R, Peritz E, Gabriel KG: On closed testing procedures with special reference to ordered analysis of variance. Biometrika 1976;63:656-660.

17 Lansberg MG, Thijs VN, Bammer R, Kemp S, Wijman CA, Marks MP, Albers GW: Risk factors of symptomatic intracerebral hemorrhage after t-PA therapy for acute stroke. Stroke 2007;38:2275-2278.

18 Hacke W, Kaste M, Fieschi C, von Kummer R, Davalos A, Meier D, Larrue V, Bluhmki E Davis S, Donnan G, Schneider D, Diez-Tejedor E, Trouillas P: Randomised double-blind placebo-controlled trial of thrombolytic therapy with intravenous alteplase in acute ischaemic stroke (ECASS II). Second European-Australasian acute stroke study investigators. Lancet 1998;352:1245-1251.

19 Wahlgren N, Ahmed N, Davalos A, Ford GA, Grond M, Hacke W, Hennerici MG, Kaste M Kuelkens S, Larrue V, Lees KR, Roine RO, Soinne L, Toni D, Vanhooren G: Thrombolysis with alteplase for acute ischaemic stroke in the safe implementation of thrombolysis in stroke-monitoring study (SITS-MOST): an observational study. Lancet 2007;369: 275-282.
20 Shaltoni HM, Albright KC, Gonzales NR, Weir RU, Khaja AM, Sugg RM, Campbell MS 3rd, Cacayorin ED, Grotta JC, Noser EA: Is intra-arterial thrombolysis safe after fulldose intravenous recombinant tissue plasminogen activator for acute ischemic stroke? Stroke 2006;38:80-84.

21 Flaherty ML, Woo D, Kissela B, Jauch E, Pancioli A, Carrozzella J, Spilker J, Sekar P, Broderick J, Tomsick T: Combined IV and intra-arterial thrombolysis for acute ischemic stroke. Neurology 2005;64:386-388.

-22 Brekenfeld C, Remonda L, Nedeltchev K, Arnold M, Mattle HP, Fischer U, Kappeler L, Schroth G: Symptomatic intracranial haemorrhage after intra-arterial thrombolysis in acute ischaemic stroke: assessment of $294 \mathrm{pa}-$ tients treated with urokinase. J Neurol Neurosurg Psychiatry 2007;78:280-285.

-23 Tanne D, Kasner SE, Demchuk AM, KorenMorag N, Hanson S, Grond M, Levine SR: Markers of increased risk of intracerebral hemorrhage after intravenous recombinant tissue plasminogen activator therapy for acute ischemic stroke in clinical practice: the multicenter rt-PA stroke survey. Circulation 2002;105:1679-1685.

24 Albers GW, Thijs VN, Wechsler L, et al: Magnetic resonance imaging profiles predict clinical response to early reperfusion: the diffusion and perfusion imaging evaluation for understanding stroke evolution (DEFUSE) study. Ann Neurol 2006;60:508517.

25 del Zoppo GJ, Higashida RT, Fulan AJ, et al: PROACT: a phase II randomized trial of recombinant pro-urokinase by direct arterial delivery in acute middle cerebral artery stroke. PROACT Investigators. Prolyse in Acute Cerebral Thromboembolism. Stroke 1998;29:4-11 